\title{
Special focus on advanced techniques for event-triggered control and estimation*
}

With the rapid development of control and estimation techniques for handling complicated system dynamics but in resource-limited microprocessors and bandwidth-limited networks, there is a demanding request for advanced event-triggered techniques. In recent years, researchers have made extensive effort on event-triggered approaches in handling various engineering problems. This special focus seeks collection of these state-of-the-art achievements in the field. It is envisioned that the contributions on advanced techniques for design of event-triggered control and estimation strategies will increase the functionality of microprocessors and efficiency of network communication in modern industrial systems.

This special focus consists of one review and eleven research papers. The review by the team of guest editors revisited some existing triggering techniques to bring readers better understanding of triggering mechanisms, the underlying technical challenges, and some promising future research topics. While state feedback control for linear systems with event-driven control is mature, output feedback control is a more challenging topic, on which Zhang J H et al. presented some new results. Consensus of multi-agent systems has become one of the most active topics. Some interesting results for event-based consensus can be found in the two papers by Ding R et al. and Li L L et al. Not only for multi-agent networks, various event-triggered techniques have been actively developed but also for neural networks; see the two papers by Lv M G et al. and Zhang Y J et al. Security of networked systems is a considerable topic over the past years; two papers in this special focus studied event-triggered control under DoS attacks. The one by Chen X L et al. is concerned with tracking control and the other by Yang Y et al. with consensus control. Nonlinear control has been one of the central research topics for several decades and it is an indispensable stage for the development of advanced event-triggered techniques. This special focus collects four papers that play on this stage: Gao Y F et al. proposed a new approach for event-triggered stabilization of general nonlinear systems, Dong L et al. studied the receding horizon principle in an event-triggered setting, Hu X D et al. investigated a new Lyapunov-based approach for handling systems subject to disturbances and delays, and $\mathrm{Ma} \mathrm{H}$ et al. designed an adaptive control scheme also for systems with disturbances. Overall, we expect that the collection of these new results would bring researchers a feast of advanced event-triggered control and estimation techniques, and foster the growth of this area to a new stage.

Finally, we would like to sincerely appreciate all the authors for their contributions to this special focus. Our appreciation also goes to all the anonymous reviewers for their voluntary and professional services during the whole review process. Last but not least, we thank SCIENCE CHINA Information Sciences Editorial Office for the invaluable supports that turn the idea of this special focus into reality.

Guest Editors:

Zhiyong CHEN, University of Newcastle, Australia

Qing-Long HAN, Swinburne University of Technology, Australia

Zheng-Guang WU, Zhejiang University, China

Yamin YAN, University of Newcastle, Australia

*Citation Chen Z Y, Han Q-L, Wu Z-G, et al. Special focus on advanced techniques for event-triggered control and estimation. Sci China Inf Sci, 2020, 63(5): 150200, https://doi.org/10.1007/s11432-019-2703-3 